\title{
Lipoma of the Stomach Prolapsing into the Duodenal Bulb and Causing a Duodenal Ulcer: A Case Report
}

\author{
Tateki Yamane ${ }^{1}$, Akihiko Takeda ${ }^{1}$, Takayuki Ishii ${ }^{2}$, Toshio Morisane ${ }^{1}$ \\ ${ }^{1}$ The International University of Health and Welfare, Shioya Hospital, Yaita, Tochigi, Japan; ${ }^{2}$ Sanikukai Family Clinic, Funabashi, \\ Chiba, Japan. \\ Email: yamane@iuhw.ac.jp
}

Received April $8^{\text {th }}, 2011$; revised May 13 ${ }^{\text {th }}, 2011$; accepted July 20 ${ }^{\text {th }}, 2011$.

\begin{abstract}
We report a case of lipoma in the antum of the stomach which prolapsed into the duodenal bulb and caused a duodenal ulcer, speculated to have been induced by the friction of its top against the duodenal mucosa. Although the ulcer healed after the administration of a proton pump inhibitor, the symptom of epigastric discomfort continued, suggested to be due to prolapsing. Therefore, a laparoscopic operation was conducted. The incidence of lipoma of the stomach is rare, and cases of its prolapse into the duodenum are very few. Furthermore, it is extremely rare for it to cause a duodenal ulcer. Because these features made this case clinically interesting, we report it here.
\end{abstract}

Keywords: Gastric Lipoma, Prolapse, Duodenal Ulcer

\section{Introduction}

Lipoma of the stomach is unusual in gastric tumors [1]. And it rarely prolapses into the duodenal bulb. We report an extremely rare case of lipoma in the antrum of the stomach which prolapsed into the duodenal bulb, leading to the development of a duodenal ulcer, speculated to have been caused by the friction of its head against the duodenal mucosa. In this case, although the duodenal ulcer scarred after the administration of a proton pump inhibitor, the symptom of epigastric discomfort continued, suggested to be due to prolapsing. So the lipoma was resected on a laparoscopic operation. After the operation, her symptom disappeared and the duodenal ulcer maintained scarring status without dosage of gastric acid inhibitor.

\section{Case Report}

An 84-year-old woman visited our hospital because of epigastric pain and discomfort of 3 months' duration. She took hypotensive drugs for hypertension, but never NSAIDs. She had a small physique and had no fever, anemia, or jaundice. Laboratory tests showed normal blood cell counts, a normal blood chemistry, and no inflammatory reactions. Serum anti-Helicobacter pylori (Hp) antibody was negative, and the serum gastrin level measured later was within normal limits. On gastrointestinal endoscopy, a 35-mm submucosal tumor (SMT) with erosions in part was observed on the anterior wall in the antrum of the stomach, prolapsing through the pylorus into the duodenal bulb (Figure 1(a)). The cushion sign of the SMT was positive (Figure 1(b)). The top of the SMT, prolapsing into the duodenal bulb, was near the posterior wall of the duodenal bulb, and a huge ulcer was detected at the same site of the duodenal bulb (Figure 1(c)). The SMT was pulled back from the pylorus with biopsy forceps, but it prolapsed easily into the duodenal bulb. On endoscopic ultrasonography (EUS), the SMT was indicated as a hyperechoic mass arising from the submucosal layer (Figure 2(a)). On a CT scan of the abdomen, about 30-mm low-density mass was observed in the antrum of the stomach.

The SMT was considered to be a lipoma because of cushion sign-positivity and the CT and EUS findings. A proton pump inhibitor was prescribed for the duodenal ulcer. Although the ulcer had scarred on repeated gastrointestinal endoscopy six weeks later (Figure 2(b)), epigastric discomfort continued, suggested to be due to prolapsing. At first, we examined whether or not endoscopic mucosal resection for the SMT was possible or not, but got it up because the base of the lipoma was 


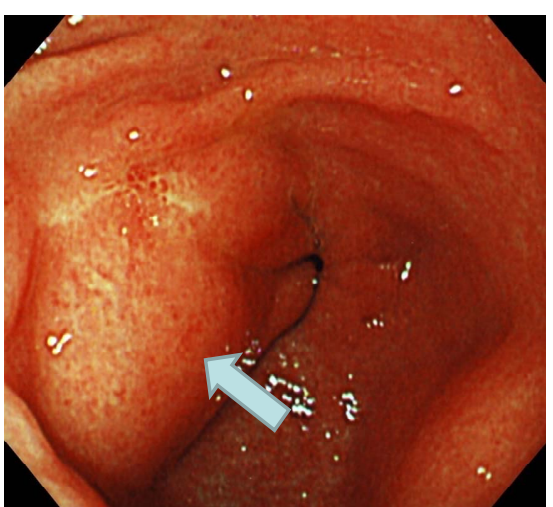

(a)

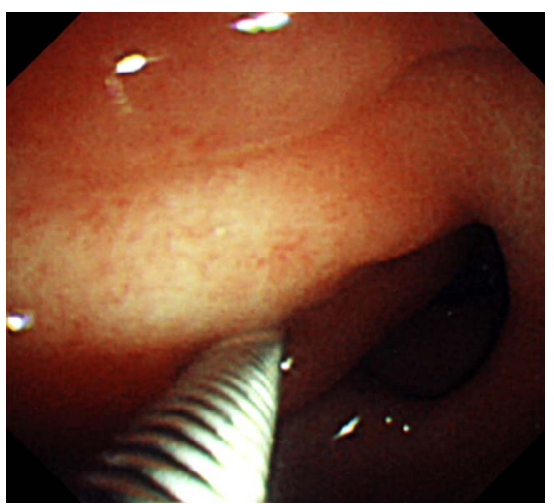

(b)

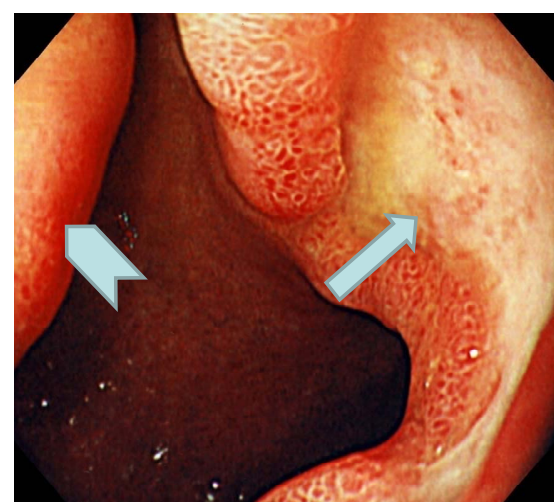

(c)

Figure 1. Initial gastrointestinal endoscopic findings. (a) An SMT was observed in the antrum of the stomach, prolapsing into the duodenal bulb (arrow). (b) Cushion sign of the SMT was positive. (c) The top of the SMT was near the posterior wall of the duodenal bulb (arrow head), and a huge ulcer was detected at the same site as the duodenal bulb (arrow).

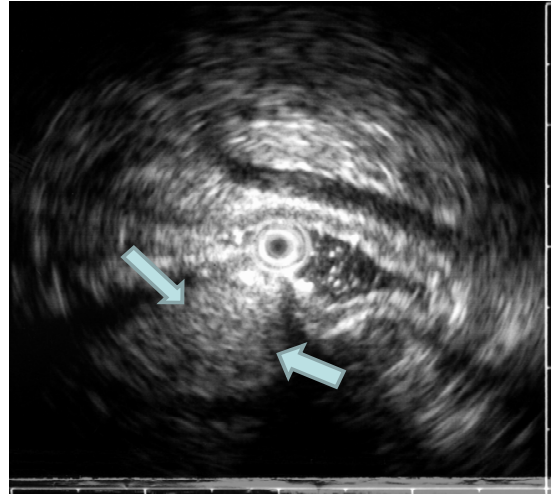

(a)

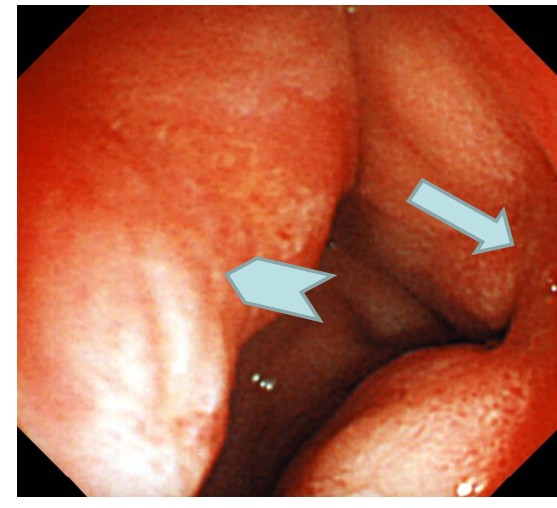

(b)

Figure 2. EUS and repeated gastrointestinal endoscopic findings. (a) The SMT was identified as a hyperechoic mass arising from the submucosal layer on EUS (arrows). (b) The top of the SMT was still erosive (arrow head) but the duodenal ulcer had scarred arrow), on repeated gastrointestinal endoscopy.

wide and its stalk was thick. Therefore, a laparoscopic operation was performed: the lipoma was tracted out of the stomach through an incision made in the anterior wall near the lesser curvature of the antrum, and it was resected. The resected specimen was $3.5 \times 3.0 \mathrm{~cm}$, and a subepithelial part of it showed a yellowish color (Figure 3(a)). The tumor was definitely diagnosed as a lipoma because the proliferation of mature fat cells was detected in the submucosa histologically (Figures 3(b) and (c)). After the operation, her symptom improved and the duodenal ulcer maintained scar status without administration of gastric acid inhibitor.

\section{Discussion}

Lipoma of the digestive tract is a benign tumor in which the proliferation of mature fat cells is observed in the submucosae histologically. Small-sized lipomas are mostly asymptomatic, are incidentally detected on endoscopic examination or contrasting X-ray study of the digestive tract. However, large-sized lipomas occasionally cause abdominal pain, gastrointestinal bleeding, or ileus due to their invagination.

Mayo et al. [1] reported that the incidence of lipoma of the alimentary tract is $4 \%$ on the examination of 4,000 surgically resected benign tumors of the digestive tract. They also stated that lipomas are most commonly found in the large intestine (64\%), followed by the duodenum/ small intestine (31.2\%), stomach (3.2\%), and esophagus (1.6\%) [1]. Thus, lipomas rarely occur in the stomach. It has been pointed out that lipoma of the stomach often occurs in the antrum [2,3], as observed in the present case. It has been also reported that lipoma of digestive tract often shows a yellowish color but it ordinarily shows a normal color in the stomach because the epithelium of the stomach is thick [3], as also found in this case. Further, it has been reported that lipoma of the stomach sometimes causes gastrointestinal bleeding because of friction against the surrounding mucosa and the influence 


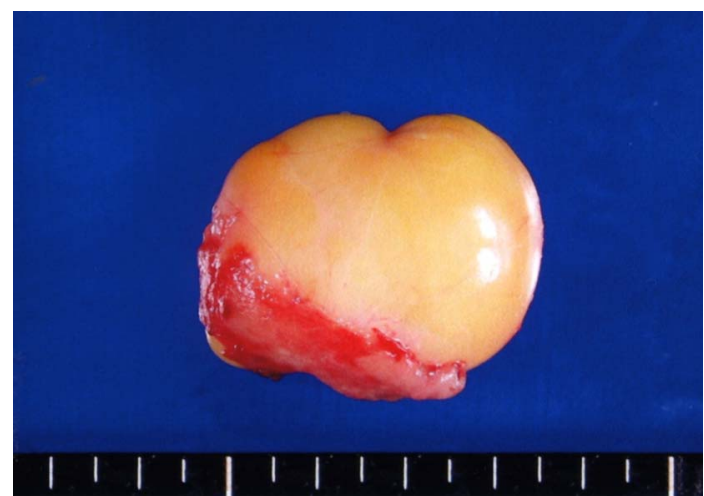

(a)

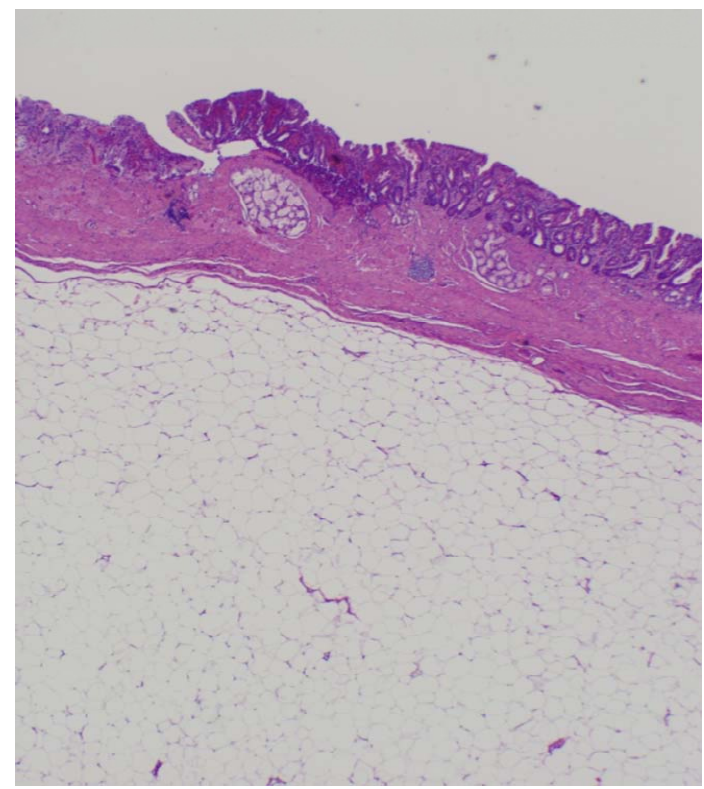

(b)

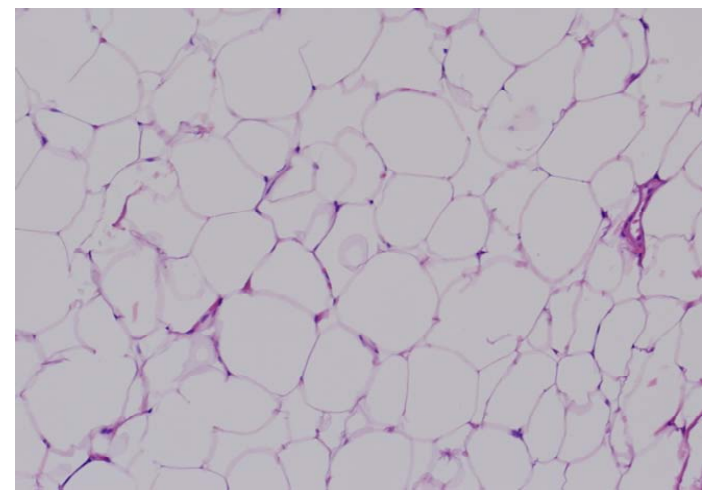

(c)

Figure 3. Finding of the resected specimen. (a) The resected specimen was $3.5 \times 3.0 \mathrm{~cm}$, and its subepithelial part showed a yellowish color. (b) and (c) The proliferation of mature fat cells was detected in the submucosa histologically (HE staining, (b) low-power view, (c) high-power view). of gastric acid [4]. However, in the present case, the lipoma itself showed no gastrointestinal bleeding, although erosions were found on its surface.

Reportedly, gastric tumors rarely prolapse into the duodenum [5-7]. Also, in prolapsing gastric tumors, it has been reported that early gastric cancer is the most common, followed by advanced gastric cancer, GIST, and lipoma, which is rare [5-7]. A MEDLINE and Japan Central Revuo Medicina-based search of the literature published between 1950 and 2008 using the key words "lipoma", "stomach", and "prolapse" revealed only 7 similar Japanese cases [5-7]. These 8 cases including the present case showed a predilection for female (females: 6 , males: 2) [5-7]. Also these patients ranged in age from 46 to 91 years with a mean of 68.3 years [5-7], suggesting a predilection for the aged. Regarding symptoms, nausea/ vomiting or epigastric pain/discomfort were observed in 5 , melena in 1 , and no symptoms in 1 [5-7]. In all cases, lipomas were found in the antrum. These 8 lipomas ranged in size from 1.9 to $6.0 \mathrm{~cm}$, and concerning the form, the semi-peduncular type comprised 7 and the sessile type comprised 1 [5-7]. As for treatment, endoscopic polypectomy was performed in 2 and surgical operation in 6 [5-7]. Laparoscopic operation was performed only in the present case. A preoperative diagnosis of lipoma was possible in 3 [5-7]. The so-called "ball valve syndrome (BVS)" was defined by Hobbs et al. [8], in which severe abdominal pain, vomiting or abdominal fullness occurs suddenly due to the prolapsing of gastric tumors or mucosae into the duodenum and blocking of the pylorus. In these 8 cases, BVS was noted in 3 [5-7]. In the present case, BVS was denied because the symptoms were neither severe nor sudden. It has been reported that the prolapsing of gastric tumors requires the essence of several conditions, as follows [9]: the tumors must be peduncular or semi-peduncular forms, they must be located on the greater curvature or anterior wall, they must show mobility and be of a sufficient weight, and peristalsis or relaxation of the pylorus must exist to push out the tumors. Through an analysis of the reported cases, prolapse was mainly observed in small and aged females. The present case corresponded well with these conditions and features.

In this case, it was extremely peculiar for a duodenal ulcer to be detected on the posterior wall of the duodenal bulb near the top of the lipoma. The patient had no history of duodenal ulcer, had never taken NSAIDs, nor had been infected by Hp. Her serum gastrin level was also within normal limits. And after the resection of the lipoma, the ulcer maintained scarring status without gastric acid inhibitor. Therefore, regarding the genesis of this duodenal ulcer, it was speculated that the duodenal mucosa had rubbed against the top of the lipoma, causing 
the ulcer. Furthermore, because the ulcer had scarred after the administration of the PPI, it was also speculated that gastric acid had advanced the erosions caused by rubbing to the ulcer. Based on our search, up to the present, there has been no report on gastric tumors, comprising lipomas, prolapsing into the duodenum and causing a duodenal ulcer, as in this case.

Thus, the present case was rare. In this case, the lipoma always showed prolapse in all endoscopic examinations, suggesting that the top of the lipoma frequently rubbed against the duodenal mucosa. Further, the top of the lipoma was movable in the duodenal bulb, suggesting that it easily rubbed against the duodenal mucosa. And it was also speculated as one of the reasons that the duodenal mucosa is thin and weak comparing with the antral mucosa covered the surface of the lipoma.

\section{Conclusions}

We reported a rare case of lipoma of the stomach which prolapsed into the duodenal bulb, leading to the development of a duodenal ulcer, speculated to have been induced by the friction.

\section{REFERENCES}

[1] C. W. Mayo, R. J. G. Pagtalunan and D. J. Brown, "Lipoma of the Alimentary Tract,” Surgery, Vol. 53, 1963, pp. 598-603.

[2] H. Kozawa, K. Momma, M. Yoshida, et al., "Endoscopic Diagnosis of Gastrointestinal Submucosal Tumor Differen- tial Diagnosis of Submucosal Tumor of the Upper Gastrointestinal Tract by Endoscopy,” in Japanese, Stomach and Intestine, Vol. 39, No. 4, 2004, pp. 446-456.

[3] I. Hirata, E. Umegaki, K. Hayashi, et al., "Diagnosis and Treatment of Lipoma in the Alimentary Tract," in Japanese, Stomach and Intestine, Vol. 39, No. 4, 2004, pp. 601-611.

[4] E. D. Palmer, "Benign Intramural Tumors of the Stomach,” Medicine, Vol. 30, No. 4, 1951, pp. 81-181.

[5] H. Machishi, M. Suzaki, T. Mutoh, et al., "A Case of Gastric Lipoma Prolapsed into the Duodenal Bulb, Which Caused Ball Valve Syndrome,” in Japanese, Japanese Journal of Gastroenterology, Vol. 93, No. 8, 1996, pp. 560-564.

[6] S. Yazumi, H. Nakase, Y. Matsushima, et al., "The 'Scarf-Ring Sign' of Ball Valve Syndrome,” Gastrointestinal Endoscopy, Vol. 55, No. 4, 2002, p. 560.

[7] T. Hori, Y. Okada, H. Machishi, et al., "A Case of Gastric Gastrointestinal Autonomic Nerve Tumor of Fornix Prolapsed into Bulbus of the Duodenum with Ball Valve Syndrome,” in Japanese, Japanese Journal of Gastroenterology, Vol. 100, 2003, pp. 673-679.

[8] W. H. Hobbs and S. E. Cohen, "Gastroduodenal Invagination Due to a Submucous Lipoma of the Stomach," The American Journal of Surgery, Vol. 71, No. 4, 1946, pp. 505-518. doi:10.1016/0002-9610(46)90199-7

[9] T. Yoshida, T. Masunaga, T. Taguchi, et al., “A Case Report of Gastroduodenal Intussusception Caused by a Leiomyoma Arising from Gastric Body (So-Called Ball Valve Syndrome)," in Japanese, Japanese Journal of Gastroenterology, Vol. 91, 1994, pp. 1228-1233. 